\title{
Performance Analysis of the Atlantis Intake System
}

\author{
Steven J. Wilson* and Craig T. Johansen ${ }^{\dagger}$ \\ University of Calgary, Calgary, Alberta, T2N 1N4, Canada \\ Vladimir Mravcak $\ddagger$ \\ Atlantis Research Labs Inc., Regina, Saskatchewan, S4W 1A3, Canada
}

\begin{abstract}
A control volume analysis of a novel dynamic intake system known as the Atlantis Intake System (AIS) used to supply air and fuel to a ramjet engine has been performed. The AIS, being developed by Atlantis Research Labs, is based on the work of Eugene Gluhareff. Since Gluhareff's pressure jet design was originally intended for tip propulsion of helicopter rotor blades, it has been modified to work with a ramjet engine. The AIS utilizes three inlet stages where an under-expanded fuel jet is injected to draw in ambient air, producing a high velocity reactive mixture. Deceleration in a supersonic diffuser results in a high stagnation pressure gas mixture entering the combustion chamber. A method for the prediction of downstream mixture properties and resulting engine performance is derived for both static and dynamic operation. Performance is assessed in terms of specific impulse. The prediction models are validated against numerical simulations that replicate flow conditions associated with the AIS. Means of entrainment are studied through an analysis of the numerical simulation results.
\end{abstract}

\section{Nomenclature}

$\begin{array}{ll}A & \text { Area, } \mathrm{m}^{2} \\ A R & \text { AIS area ratio (Outlet/Inlet) } \\ C_{P} & \text { Specific heat capacity, J/g K } \\ \dot{E} & \text { Energy flow rate, } \mathrm{J} / \mathrm{s} \\ E R & \text { Equivalence ratio } \\ F_{T} & \text { Force of Thrust, } \mathrm{N} \\ I_{s p} & \text { Specfic impulse, } \mathrm{s} \\ J P R & \text { Jet pressure Ratio } \\ M & \text { Molar mass, mol/g } \\ M a & \text { Mach number } \\ M A F & \text { Air-to-Fuel mass flow ratio } \\ M A F_{\text {stoichiometric }} & \text { Stoichiometric air-to-fuel mass flow ratio } \\ \dot{m} & \text { Mass flow rate, } \mathrm{kg} / \mathrm{s} \\ \dot{P} & \text { Momentum flow rate, } \mathrm{N} \\ p & \text { Pressure, Pa } \\ R & \text { Gas constant, } \mathrm{J} / \mathrm{g} \mathrm{K} \\ R_{u} & \text { Univesal gas constant, } \mathrm{J} / \mathrm{mol} \mathrm{K} \\ T & \text { Temperature, } \mathrm{K} \\ T_{\text {max }} & \text { Maximum temperature reached in combustion chamber, } \mathrm{K} \\ U & \text { Velocity, m/s } \\ \gamma & \text { Ratio of specific heats } \\ \rho & \text { Density, kg/m }{ }^{3}\end{array}$

*MSc Student, Department of Mechanical and Manufacturing Engineering, AIAA Student Member.

${ }^{\dagger}$ Assistant Professor, Department of and Manufacturing Mechanical Engineering, AIAA Member.

${ }^{\ddagger}$ Chairman and Chief Executive Officer. 


$\begin{array}{ll}\text { Subscript } & \\ a & \text { Air property } \\ C V & \text { Control Volume } \\ f & \text { Fuel property } \\ m & \text { Mixture property } \\ 0 & \text { Stagnation property }\end{array}$

\section{Introduction}

The ramjet is one of the simplest jet engine designs as it has no moving parts and can be efficient when operating at supersonic flight speeds. However, its inability to operate statically makes its applicability limited. Often a more traditional engine, such as a turbojet, is required to accelerate the vehicle until a speed is reached where the ramjet can provide the aerodynamic compression necessary to produce sufficient thrust to maintain speed. Meaning an aircraft must carry multiple engines, which do not operate simultaneously. The Atlantis Research Labs Intake System (AIS) provides a means for introducing the airflow necessary to operate the ramjet statically without the introduction of any moving parts. Atlantis Research Labs based the design of its AIS off of the Gluhareff pressure jet engine, which was designed and built by Eugene M. Gluhareff in the 1950s for tip propulsion on rotary wing aircraft. ${ }^{1}$ The AIS uses an under expanded fuel jet injected through three inlet stages to entrain ambient air, acting as multi-stage ejector pump. The fuel, traditionally propane due to its high vapour pressure at room temperature, is preheated through a heat exchanger in the combustion chamber. A fuel jet is injected into the intake system as a grossly under-expanded jet producing oscillating positive and negative pressure nodes along the path of the jet. The viscous interactions between the fuel jet and the air, along with the interaction between the fuel jet and the stages of the inlet, draw in surrounding air producing a combustible mixture. While some experimental studies of the AIS and Gluhareff systems have been conducted analyzing performance in terms of total thrust, the theoretical gains that may be realized by this system are still largely unstudied. ${ }^{2,3}$ Determining the feasibility and range of operation is one of the primary goals of this study. Additionally, the results of this analysis will be compared to some simplified computational fluid dynamic (CFD) simulations as a means of validating the method outlined. The CFD results will also provide a means of assessing the causes of entrainment being utilized by the AIS.

The AIS is unique in that it is open to the atmosphere, though it shares many similarities with the traditional ejector pump. Models based off of the control volume analysis of an ejector pump or other mixing stream scenarios are quite common, however the operating conditions and primary goals are typically different than those of the AIS. ${ }^{4,5,6,7}$ Bernstein (1953) presented a one-dimensional analysis on parallel jet mixing encountered in the testing of engines in a supersonic wind tunnel. A model was defined which based on the mixing of two streams of the same gas allowed the prediction of the down stream Mach number. Relationships were also developed for the total downstream pressure, though this was limited to cases of equal inlet Mach number or equal inlet static temperature. Huang et al. (1999) presented a 1-D analysis using empirical coefficients to accurately predict ejector performance limited to critical mode operation, where the downstream Mach number is equal to one. John et al. (2006) provides a simple control volume analysis for a jet ejector, though it requires an iterative solution to determine the downstream properties. Liao (2008) produced a generalized model for the design of gas ejectors, though it assumed normal shock at the exit of the primary stream resulting in an equal static pressure for both streams entering the mixing chamber. This study aims to provide a simple means of predicting the overall performance of an engine utilizing the AIS for a variety of operating conditions. The formulation of an accurate model for predicting the mixture properties following the AIS will be the basis of determining overall engine performance. This model must take into consideration a variety of fuels, the static pressure ratio between the fuel and the air, the area ratio between the inlet and outlet of the AIS, and the impact of changing the velocity of the air entering the AIS.

\section{Engine Overview}

A simplified schematic of the intended engine design in use with the AIS is provided in Fig. 1. The primary focus of this study will be the section outlined in the control volume, the AIS. The simplified engine consists of a fuel tank, a heating coil used to preheat the fuel, the AIS, and an ideal engine consisting of a 
diffuser, combustion chamber and exit nozzle. In the original Gluhareff design, the propane fuel entered the heating coil as a liquid, where it was vaporized prior to injection by the heat of combustion. Through this study, it was found that altering the amount of heat transferred to pre-heat the fuel stream changed the overall performance. For each case, an optimized amount of heat transfer was determined. A parallel fuel line that bypasses the heating coil is proposed as a means of controlling this heat transfer. Conservation of energy limits the fuel stream temperature below the temperature of the products in the combustion chamber. Based on the properties within the fuel tank, the amount of heat transferred to the fuel stream, and the Mach number of the inlet jet of the AIS, the properties of the fuel injected into the AIS $\left(p_{f}, T_{f}\right.$, and $\left.U_{f}\right)$ may be determined. Based on these properties and those of the ambient surroundings, it is desired to determine the properties of the mixture that will exit the AIS and enter the diffuser of the ramjet. If these properties entering the diffuser can be determined, an ideal jet-propulsion cycle can be used to predict the overall engine performance. Additional considerations must be made if the vehicle has a finite velocity. In this case, the air mass flow rate and stagnation properties are increased, and the ability of the AIS to entrain air becomes less effective due to the low relative velocity of the fuel jet. This will be further discussed in section (V).

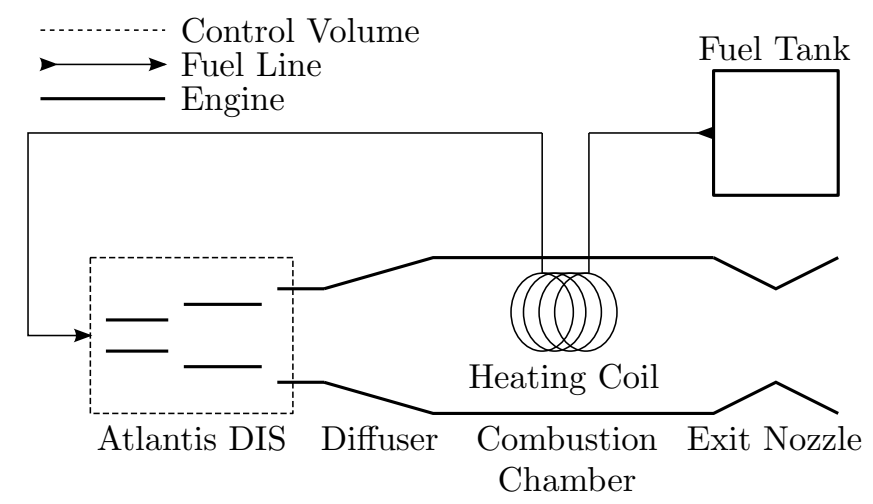

Figure 1. Simplified schematic of an ideal engine utilizing the AIS.

\section{Static Operation Control Volume Analysis}

Primarily, this work will focus on the static performance of the AIS. At zero velocity, there will be no air induced into the engine by the movement of the vehicle. In order to supply air to burn the fuel injected, all airflow must be supplied by entrainment through the use of the AIS. Assuming that the properties of the fuel jet entering the AIS and the surrounding air, as well as the relative physical dimensions of the inlet and outlet of the AIS are known, the goal is to predict the properties of the fuel-air mixture exiting the control volume into the diffuser.

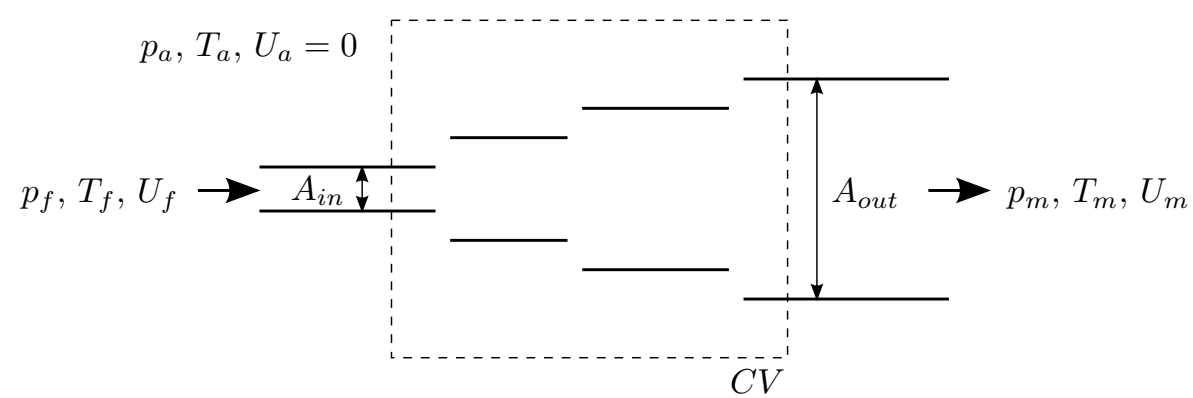

Figure 2. The control volume around the three stages of the Atlantis Intake System. Image is not to scale.

A control volume is drawn encompassing the inlet and outlet of the AIS (see Fig. 1 and 2). Some initial assumptions are necessary in order to approach the problem. It is assumed that only fuel and air enter the domain, and a mixture of the two is all that exits the domain through the outlet of the AIS. All inlet and outlet streams are at uniform properties along the boundary of the control volume, and any velocity at the 
boundaries is normal to it. Therefore it is assumed we have two uniform inlet streams, fuel and air, and one uniform outlet stream, a mixture of fuel and air. Knowing that the flux of mass, momentum and energy entering the domain must be equal to those of the outlet stream leaving the domain, we have three equations which may be solved to determine our three downstream properties $p_{m}, T_{m}$, and $U_{m}$.

Applying a conservation of mass:

$$
\dot{m}=\dot{m}_{i n}=\frac{p_{f}}{R_{f} T_{f}} A_{i n} U_{f}+\frac{p_{a}}{R_{a} T_{a}}\left(A_{C V}-A_{\text {out }}-A_{\text {in }}\right) U_{a}=\dot{m}_{\text {out }}=\frac{p_{m}}{R_{m} T_{m}} A_{\text {out }} U_{m}
$$

As the mass flow rate of air entering the domain must be finite, if the control volumes is extended infinitely in all directions the term $A_{C V}$ in Eq. (1) will approach infinity and the term $U_{a}$ must approach zero, this term then becomes undefined. Due to this we implement the relation $M A F=\dot{m}_{a} / \dot{m}_{f}$, resulting in the following:

$$
\dot{m}_{i n}=\frac{p_{f}}{R_{f} T_{f}} A_{i n} U_{f}(1+M A F)
$$

Solving Eq. (1) for $U_{m}$ :

$$
U_{m}=\frac{\dot{m} R_{m} T_{m}}{A_{\text {out }} p_{m}}
$$

Applying a conservation of X-Momentum:

$$
\dot{P}=\dot{P}_{\text {in }}=p_{f} A_{\text {in }}+p_{a}\left(A_{\text {out }}-A_{\text {in }}\right)+\dot{m}_{f} U_{f}+\dot{m}_{a} U_{a}=\dot{P}_{\text {out }}=p_{m} A_{\text {out }}+\dot{m} U_{m}
$$

It can be seen in Fig. 2 that the pressure forces due to the air on each side beyond the outlet area of the control volume are equal and opposite, resulting in the simplified term $p_{a}\left(A_{\text {out }}-A_{\text {in }}\right)$. In the case of static operation, if the control volume is extended infinitely in all directions it can be assumed that the velocity of the air entering the control volume will approach 0 . As $\dot{m}_{a}$ has a finite magnitude, it is then assumed that the term $\dot{m}_{a} U_{a}$ may be neglected.

Solving Eq. (4) for $p_{m}$ :

$$
p_{m}=\frac{\dot{P}-\dot{m} U_{m}}{A_{\text {out }}}
$$

Applying a conservation of Energy:

$$
\dot{E}=\dot{E}_{\text {in }}=\dot{m}_{f} C_{P f} T_{0 f}+\dot{m}_{a} C_{P a} T_{0 a}=\dot{E}_{\text {out }}=\dot{m} C_{P m}\left(T_{m}+\frac{U_{m}^{2}}{2 C_{P m}}\right)
$$

Solving Eq. (6) for $T_{m}$ :

$$
T_{m}=\frac{\dot{E}}{\dot{m} C_{P m}}-\frac{U_{m}^{2}}{2 C_{P m}}
$$

In the case of static operation, as the velocity of the air entering the control volume is assumed to be negligible, the static and stagnation temperature can be assumed to be equal $\left(T_{0 a}=T_{a}\right)$.

Substituting Eq. (5) and (7) into Eq. (3) and collecting the like terms of $U_{m}$ results in the following, which can be seen is in the form of a quadratic equation.

$$
\left[\dot{m}\left(1-\frac{R_{m}}{2 C_{P m}}\right)\right] U_{m}^{2}+[-\dot{P}] U_{m}+\left[\frac{R_{m} \dot{E}}{C_{P m}}\right]=0
$$


Eq. (8) can then easily be solved by putting it in the form of the quadratic formula:

$$
U_{m}=\frac{\dot{P} \pm \sqrt{\dot{P}^{2}-4\left[\dot{m}\left(1-\frac{R_{m}}{2 C_{P m}}\right)\right]\left[\frac{R_{m} \dot{E}}{C_{P m}}\right]}}{2\left[\dot{m}\left(1-\frac{R_{m}}{2 C_{P m}}\right)\right]}
$$

As the properties of the downstream mixture are unknown, the variables $\dot{P}, \dot{m}$, and $\dot{E}$ in Eq. (9) should be expressed in terms of input parameters as seen in Eq. (2), (4), and (6). Applying the relations $A R=$ $A_{\text {in }} / A_{\text {out }}, J P R=p_{f} / p_{a}, M a=U / \sqrt{\gamma R T}$, and $R / C_{P}=(\gamma-1) / \gamma$ results in the following:

$U_{m}=\frac{\left[1+\frac{A R-1}{J P R}+\gamma_{f} M a_{f}^{2}\right] \pm \sqrt{\left[1+\frac{A R-1}{J P R}+\gamma_{f} M a_{f}^{2}\right]^{2}-2\left[\frac{\gamma_{f} M a_{f}^{2}}{R_{f} T_{f}}(1+M A F)\left(\frac{\gamma_{m}^{2}-1}{\gamma_{m}^{2}}\right)\right]\left[C_{P f} T_{0 f}+C_{P a} T_{a}\right]}}{\left[\sqrt{\frac{\gamma_{f}}{R_{f} T_{f}}} M a_{f}(1+M A F)\left(\frac{\gamma_{m}+1}{\gamma_{m}}\right)\right]}$

Mass averaged specific heat capacity of the mixture: ${ }^{9}$

$$
C_{P m}=\frac{C_{P f}+M A F C_{P a}}{1+M A F}
$$

Molar mass of the mixture:

$$
M_{m}=\frac{1+M A F}{\frac{1}{M_{f}}+\frac{M A F}{M_{a}}}
$$

Specific gas constant of the mixture:

$$
R_{m}=\frac{R_{u}}{M_{m}}
$$

Ratio of specific heats of the mixture:

$$
\gamma_{m}=\frac{C_{P m}}{C_{P m}-R_{m}}
$$

From Eq. (10) it can be seen that $U_{m}=f\left(A R, J P R, \gamma_{f}, \gamma_{m}, R_{f}, C_{P f}, C_{P a}, M a_{f}, T f, T_{a}, M A F\right)$, the term $T_{0 f}$ may be expressed in terms of $\gamma_{f}$ and $M_{f}$. All of these variables are a function of either the physical design of the AIS or its input operation, with the exception of the mixture gas properties and the air-to-fuel mass flow ratio. The mixture gas properties are a function of known inlet properties based on the air-to-fuel mass flow ratio as seen in equations (11), (12), (13), and (14). Thus, the air-to-fuel mass flow ratio remains the only unknown variable. If some value of $M A F$ is assumed, $U_{m}$ can be calculated for a given array of input parameters using Eq. (10) in conjunction with equations (11), (12), (13), and (14). Once a solution to $U_{m}$ is determined, $p_{m}$ and $T_{m}$ can be determined based on equations (5) and (7), where $\dot{P}, \dot{m}$, and $\dot{E}$ can be determined based on the given inlet parameters. For static operation, the value of $M A F$ achieved by the AIS will be dependant exclusively on the amount of entrainment, however the goal will always be to achieve a design where $M A F=M A F_{\text {stoichiometric }}$.

\section{A. Ideal Jet Propulsion Cycle}

Once the properties of the mixture downstream of the AIS have been determined, the overall performance of the engine may be evaluated. First, the rest of the properties through the engine must be calculated. Following the inlet, it is assumed that the mixture is isentropically decelerated down to a negligible velocity, relative to the engine. The pressure and temperature of the mixture then rise to their appropriate stagnation properties based on isentropic relations. The mixture then passes through the combustion chamber, where it is burned at a constant pressure. This combustion is assumed to increase the stagnation temperature of the mixture, while impacts on the velocity and pressure are neglected. It may be assumed that there is a maximum temperature reached in the combustion chamber independent of input conditions due to 
dissociation. ${ }^{10}$ Finally, the exhaust gases are accelerated through a converging-diverging nozzle until the static pressure of the exhaust matches that of the atmosphere.

As the the properties determined up to this point were shown to be independent of the inlet area, and therefore the magnitude of the mass flow rate of fuel, it is useful to quantify this performance independent of these variables too. A commonly used measurement for performance of high speed air-breathing engines is specific impulse. Specific impulse quantifies thrust as a function of the amount of mass flow rate of fuel used by the engine. In the case of static operation the resulting equation is:

$$
I_{s p}=\frac{F_{T}}{\dot{m}_{f} g}=\frac{(1+M A F) U_{e x i t}}{g}
$$

The value of $U_{\text {exit }}$ may be determined based on the temperature difference of the mixture between the combustion chamber and the exit nozzle. As has been stated, the temperature of the combustion chamber will be dictated by some maximum temperature that may be achieved. Assuming the flow is isentropically accelerated to meet the static pressure of the atmosphere following the combustion chamber, the temperature at the exit can be determined by applying isentropic relations, ${ }^{9}$ resulting in:

$$
I_{s p}=\frac{(1+M A F) \sqrt{2 C_{P m} T_{\max }\left(1-\left(\frac{P_{a}}{P_{0 m}}\right)^{\frac{\gamma_{m}-1}{\gamma m}}\right)}}{g}
$$

It can be seen that the specific impulse is a function of $M A F$ and the post AIS stagnation pressure of the mixture. In the case of an engine, it is always desirable to have a stoichiometric mixture, limiting one of these parameters, although it should be noted that $M A F_{\text {stoichiometric }}$ is dependant on the fuel chosen.

\section{B. Implications of a Solution in the form of a Quadratic Equation}

It can be seen from Eq. (9) that the solution to the downstream mixture velocity is in the form of a quadratic equation. As a result, there are two important notes that should be made; first, there will always be two possible solutions for the velocity for a given array of input parameters, and second, there is a limit at which a real solution for the velocity will cease to exist (when the term under the square root of Eq. (9) is equal to 0 as seen in Eq. (17)).

$$
\dot{P}^{2}-4\left[\dot{m}\left(1-\frac{R_{m}}{2 C_{P m}}\right)\right]\left[\frac{R_{m} \dot{E}}{C_{P m}}\right]=0
$$

If this limit is expressed in terms of input parameters, the resulting inequality that must be satisfied is Eq. (18). For a typical problem, all variables will be known, with the exception of $M A F$ and $\gamma_{m}$ (which is a function of $M A F)$. In the case that the value of $M A F$ is increased, the left hand side of Eq. (18) will be decreased moving the value of $U_{m}$ towards a non-real solution. As well, typical hydrocarbons have a ratio of specific heats lower than that of air, ${ }^{11}$ increasing $M A F$ will also increase the value of $\gamma_{m}$ further moving towards a non-real solution. Therefore, for a fixed array of input parameters there exists a critical limit on $M A F$ where a real solution no longer exists.

$$
\left[1+\frac{A R-1}{J P R}+\gamma_{f} M a_{f}^{2}\right]^{2}-2\left[\frac{\gamma_{f} M a_{f}^{2}}{R_{f} T_{f}}(1+M A F)\left(\frac{\gamma_{m}^{2}-1}{\gamma_{m}^{2}}\right)\right]\left[C_{P f} T_{0 f}+C_{P a} T_{a}\right] \geq 0
$$

If Eq. (17) is expressed in terms of the downstream properties it can be simplified into the following equation, which can be seen has only one unique solution where $M a_{m}$ is equal to 1 . Therefore the limit at which a real solution ceases to exist is always when the downstream Mach number is equal to one.

$$
M a_{m}^{4}-2 M a_{m}^{2}+1=0
$$

As the limit of the real solution represents the point where $M a_{m}=1$, and the term $\dot{P}$ is independent of $M A F$, it can then be deduced from Eq. (10) that the double solution of $U_{m}$ must represent a supersonic and 
subsonic solution. This imposes the condition that for any valid combination of input parameters there are two possible solutions. The solution that is more physically accurate given the inlet conditions must then be determined. A similar analysis on the mixing of two gas streams was presented by Bernstein (1953), likewise a double valued solution for downstream Mach number was found. In order to determine which of the two solutions is correct for any case at hand, three distinct cases were presented: ${ }^{8}$

(a) In the case where both mixing streams are subsonic, obviously the subsonic solution is the only valid case as the supersonic solutions solution would represent a net decrease in entropy.

(b) In the case where both mixing streams are supersonic both solutions are physically possible. The supersonic solution will occur when the back pressure (static pressure after mixing) is low. The subsonic solution will occur when the back pressure is high, this indicates shocks will exist in the mixing region.

(c) In the case where one stream is supersonic, and one subsonic, the subsonic solution is always possible. The supersonic solution is only valid if the inlet area of the supersonic stream is significantly greater than that of the subsonic region.

This study is primarily focused on case (c) where the air contributing to the mixture is initially at rest. It is also assumed that the area of the air entering the engine is significantly greater than that of the fuel, therefore the subsonic solution is the only valid solution. Section V considers the case where the vehicle is moving, and the air entering the engine has a relative velocity. In this case as long as the vehicle is travelling at a subsonic velocity this still represents case (c), and the subsonic solution is considered to be the valid solution. In the case where the vehicle has a supersonic velocity, this represents case (b), the supersonic solution is then considered to be the valid solution as it is assumed that the back pressure is sufficiently low such that there are no shocks formed within the mixing region.

\section{Comparison to CFD}

As there is limited experimental data available for either the AIS or the Gluhareff pressure jet, some simplified CFD simulations were conducted to provide a means for comparison for the analytical solution presented above. 17 cases of the static operation of the AIS using different inlet parameters were conducted using the open source software OpenFOAM. A modified version of the native OpenFOAM solver rhoCentralFoam was used for the multi-species, compressible simulations of a methane fuel jet injected through the AIS into ambient air. ${ }^{12}$ Figure 3 shows a schematic of the computational domain and the boundary conditions. The stagnation pressure and temperature of the air at the boundary were fixed, as well as the static pressure, temperature and velocity of the fuel inlet jet. Three intake stages are modelled as infinitely thin slip boundaries in order to simplify the simulation, while providing insight into the interaction of the AIS walls and the pressure waves developed. The mesh is axi-symmetric and has been created in the native OpenFOAM meshing utility blockMesh. The computational domain has been extended radially, upstream, and downstream of the intake system to avoid potential negative interference of the region of interest with the boundary conditions. Transition to turbulence in under-expanded jets may be determined based on rescaled Reynolds number, ${ }^{13}$ though as the prediction method proposed is for an idealized case no turbulence modelling was employed. The simulations also use constant specific heat capacity and ratio of specific heats, similar to the model.

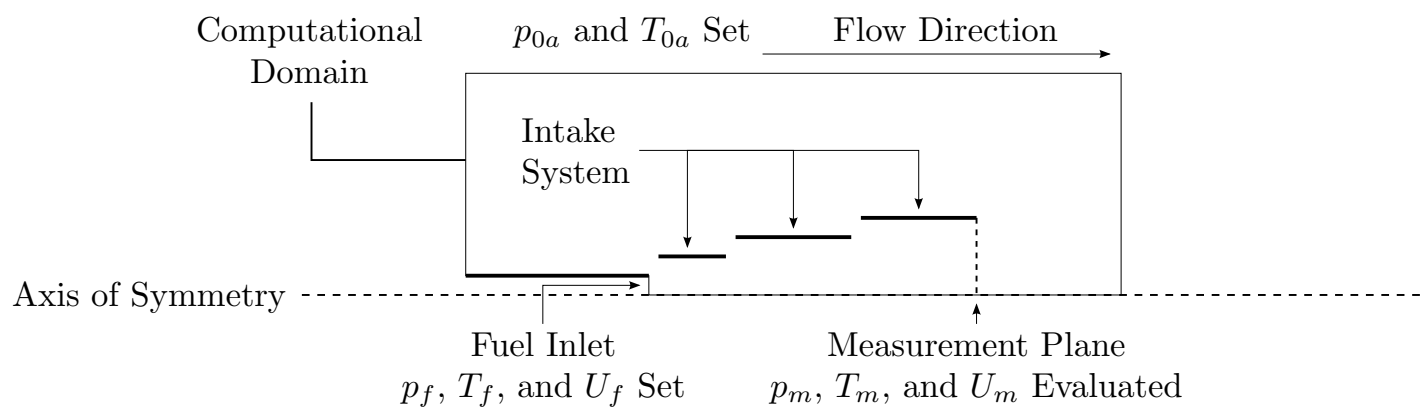

Figure 3. A schematic of the computational domain used for the AIS simulations. Initial boundary conditions and points of measurement are shown. Figure is not to scale. 
Mixture properties were evaluated at the exit of the third stage, as seen in Fig. 3 by evaluating $\dot{m}, \dot{P}$, and $\dot{E}$ at each cell along the exit plane. These values were then summed to determine the total mass, momentum, and energy exiting the AIS. Based on these total values, $p_{m}, T_{m}$, and $U_{m}$ were determined by rearranging and iteratively solving equations (1), (4), and (6) until a converged solution is determined. Figures 4 and 5 provide a comparison of mixture stagnation properties between these converged solutions and the predictions determined by the method outlined.

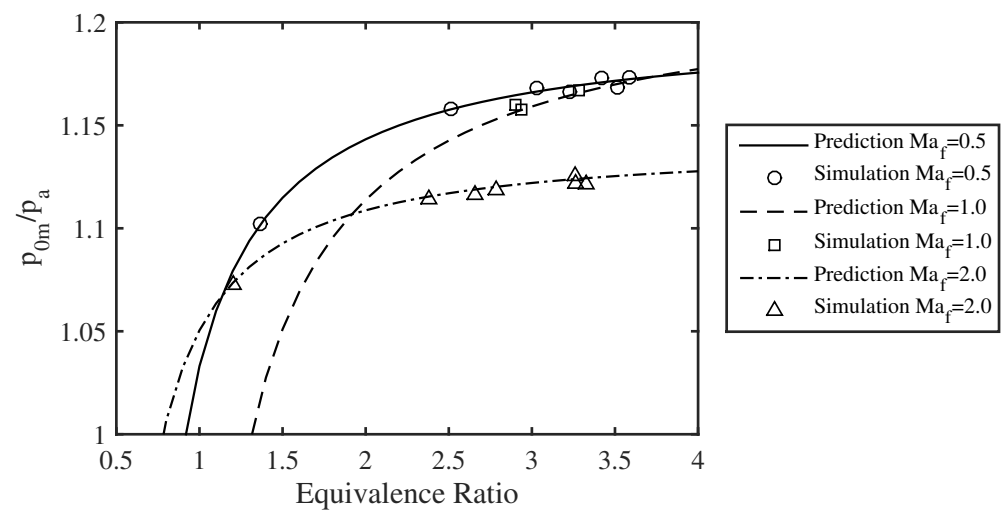

Figure 4. A comparison of exiting mixture stagnation pressure predicted with the method outlined and CFD simulations using the software OpenFOAM for different fuel jet Mach numbers, all values are normalized by the ambient air pressure.

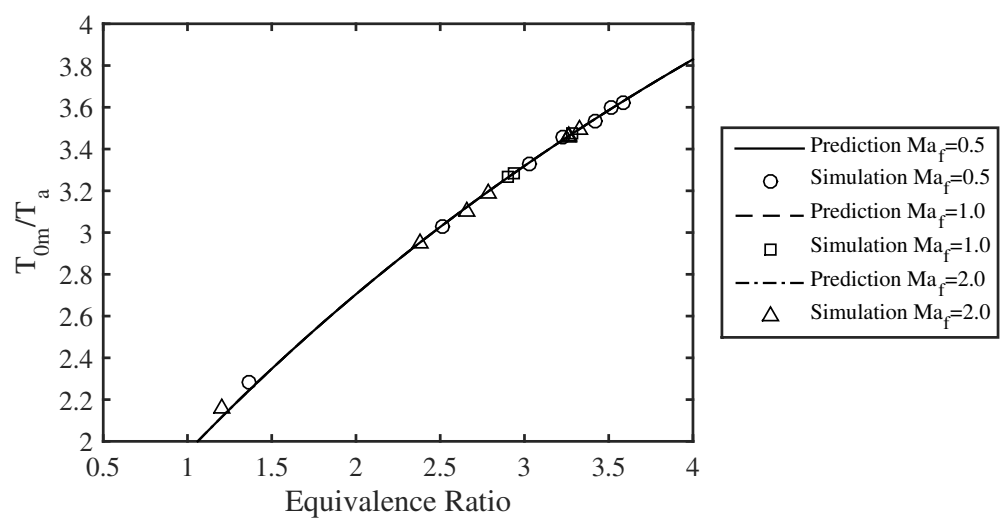

Figure 5. A comparison of exiting mixture stagnation temperature predicted with the method outlined and CFD simulations using the software OpenFOAM for different fuel jet Mach numbers, all values are normalized by the ambient air temperature.

Both the predictions and the simulations correspond to a Methane jet with fuel supplied at the same stagnation properties. The inlet conditions for all of these simulations were a methane fuel jet, with an area ratio of 250 , a jet pressure ratio of 5.92 , a fuel tank stagnation temperature of $298.15 \mathrm{~K}$, and 10 percent of the heat of combustion used to pre-heat the fuel. The different simulations represent a variation in jet Mach number as seen by the different curves. As well, the jet Reynolds's number and axial dimensions were varied for each Mach number, while this does not have any impact on the prediction, it will affect the equivalence Ratio achieved by the system. ${ }^{14}$ Both plots are normalized by the ambient air properties, and plotted against equivalence ratio $(E R)$, where $E R=M A F_{\text {stoichiometric }} / M A F$. Excellent agreement can be seen for all simulations versus the prediction model proposed. It can be seen from Eq. (6) that the stagnation temperature of the mixture is independent of the fuel jet Mach number.

\section{A. Analysis of CFD Results}

The results of the CFD simulations were assessed for determining the means of entrainment being utilized by the AIS. It is believed that the Gluhareff pressure jet used standing pressure waves to induce airflow, using a local low pressure region located at the end of each inlet stage to draw in ambient air. ${ }^{2}$ It is also well known that an under-expanded jet may entrain significant amounts of air without any interaction with 
a solid body through viscous interactions between the gases. ${ }^{14}$ Figures 6 and 7 show contours of the pressure and Y-velocity between the second and third inlet stages for one of the simulations conducted, where the positive Y-direction in the images is from the bottom going upwards. Both images have been scaled so that the smaller disturbances may be seen, the actual pressure and velocity exceed the minimum and maximums indicated by the scales.

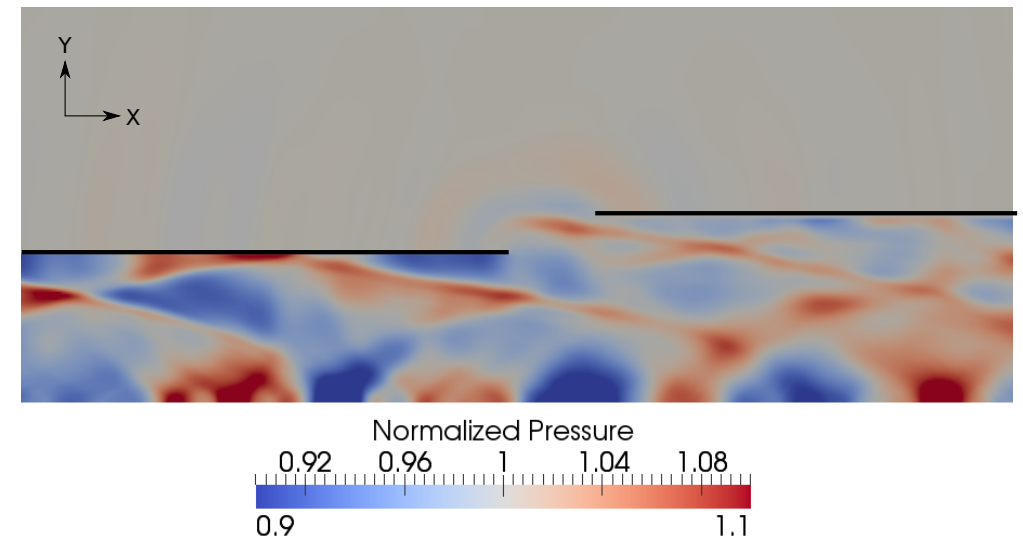

Figure 6. A contour of static pressure, normalized by the ambient air pressure, for one of the simulations conducted for the AIS. The region shown represents the break between the second and third inlet stage. The simulation uses a Methane fuel jet, $J P R=5.91$, and $M a_{f}=1.0$.

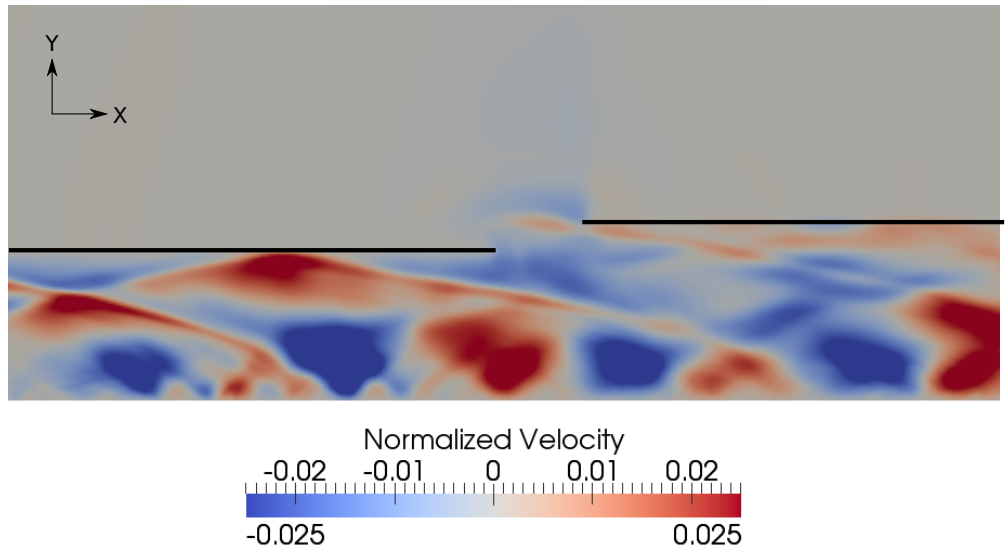

Figure 7. A contour of Y-velocity, normalized by the inlet fuel jet velocity, for one of the simulations conducted for the AIS. The region shown represents the break between the second and third inlet stage. The simulation uses a Methane fuel jet, $J P R=5.91$, and $M a_{f}=1.0$.

Figure 6 shows the pressure between the two inlet stages. It can be seen that there are local high and low pressure areas. One of the low pressure areas can be seen at the end of the second stage, and the local pressure in this region is significantly lower than that of the ambient surroundings. Figure 7 shows the velocity for the same region and time, it can be seen that above the break between the two stages, there is a significant area of negative Y-velocity. This indicates that the ambient air in the immediate surrounding area is moving downwards, towards the axis of the jet. This would indicate that there is some merit to the claim of low pressure nodes inducing airflow.

It should also be noted there a number of simulations were conducted for the same inlet conditions, but with different axial dimensions. It was found that a greater axial length resulted in a greater value of $M A F$ achieved, though the number of inlet stages remained at three. As a greater length represents more time and distance for mixing to occur, this would indicate that the viscous interactions also contribute to the overall entrainment. It is likely that both means contribute to entrainment, though it is difficult to quantify which may be the dominant means. 


\section{Dynamic Operation Performance Evaluation}

In a case where the engine has a forward velocity, some amount of air will be forced into the inlet of the ramjet (outlet of the AIS). There will exist a point where the air induced into the engine from the velocity of the vehicle surpasses the stoichiometric limit for the fuel supplied. At this point, it becomes necessary to increase the amount of fuel supplied to the engine in order to maintain the stoichiometric air-to-fuel ratio. Beyond this point, the mass flow rate of the air induced will increase proportional to the increase in velocity. It is physically unrealistic to alter the mass flow rate for the inlet jet across a suitable range and at such a rate. Therefore, for this study it is assumed that a secondary fuel inlet following the diffuser supplements the inlet jet, while all other properties specified remain constant. For the sake of the dynamic analysis, it is assumed that as the velocity of the vehicle increases, the entrainment of the jet decreases at such a rate that a stoichiometric mixture is maintained. Beyond the limit where the mass flow rate of air induced exceeds the stoichiometric limit for the inlet jet, the value of $M A F$ is then dictated by the amount of air forced into the engine inlet by its velocity and the constant mass flow rate of fuel from the inlet jet as seen in Eq. (20).

$$
M A F\left(U_{a}\right)=\left\{\begin{array}{cll}
M A F_{\text {stoichiometric }} & \text { for } \quad \rho_{a}(A R-1) U_{a} \leq M A F_{\text {stoichiometric }} \rho_{f} U_{f} \\
\frac{\rho_{a}(A R-1) U_{a}}{\rho_{f} U_{f}} & \text { for } \quad \rho_{a}(A R-1) U_{a}>M A F_{\text {stoichiometric }} \rho_{f} U_{f}
\end{array}\right.
$$

\section{A. Specific Impulse}

Similar to the case of static operation, the velocity of the resulting mixture can be determined explicitly. Equation (10) is slightly modified to incorporate the momentum term from the velocity of the air, and the increase in total temperature of the air caused by the velocity of the vehicle. The specific impulse may then be calculated, Eq. (16) is modified to include the force of the air entering the engine. Specific impulse is an important measurement for air-breathing engines as it varies drastically with vehicle velocity.

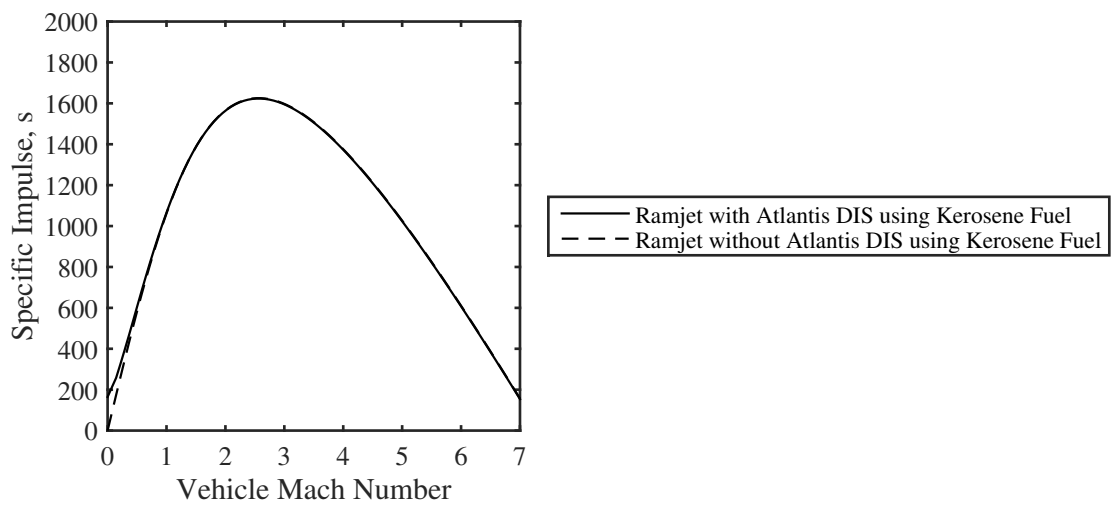

Figure 8. A comparison of Specific Impulse predicted with the method outlined for a moving vehicle with and without the AIS using a Kerosene fuel jet, $J P R=1.01, M a_{f}=2.0$, and $E R=1$.

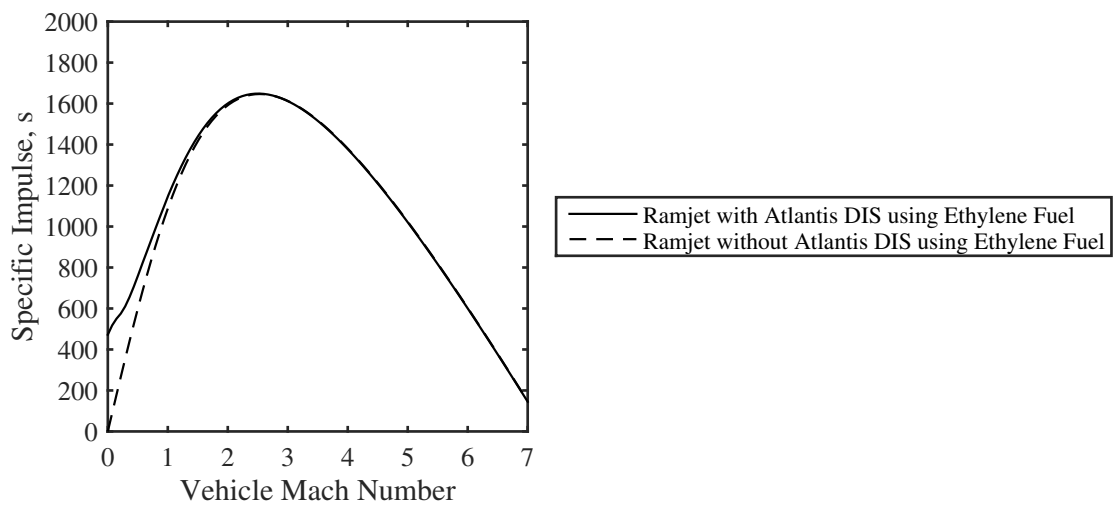

Figure 9. A comparison of Specific Impulse predicted with the method outlined for a moving vehicle with and without the AIS using a Ethylene fuel jet, $J P R=4.63, M a_{f}=2.0$, and $E R=1$. 


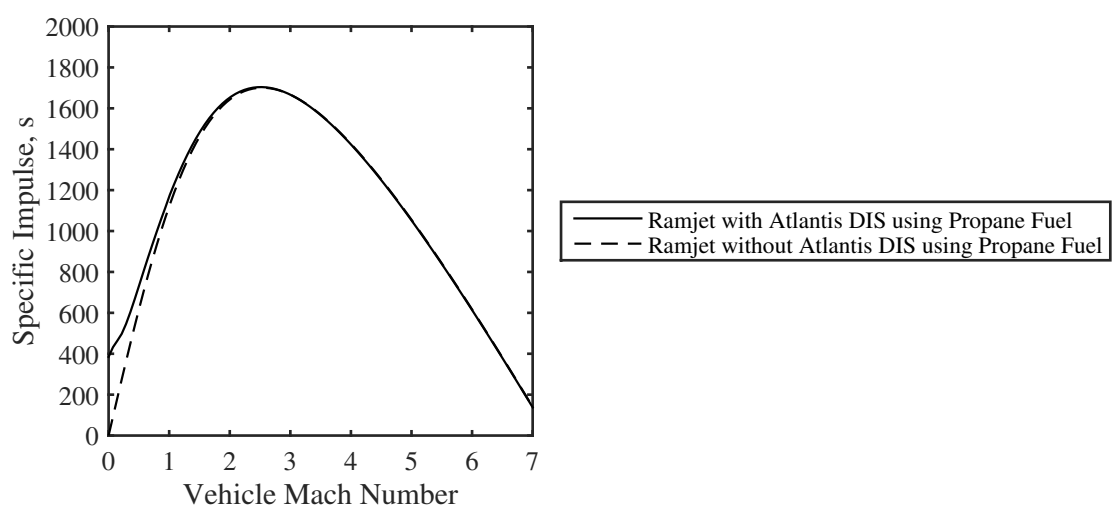

Figure 10. A comparison of Specific Impulse predicted with the method outlined for a moving vehicle with and without the AIS using a Propane fuel jet, $J P R=3.22, M a_{f}=2.0$, and $E R=1$.

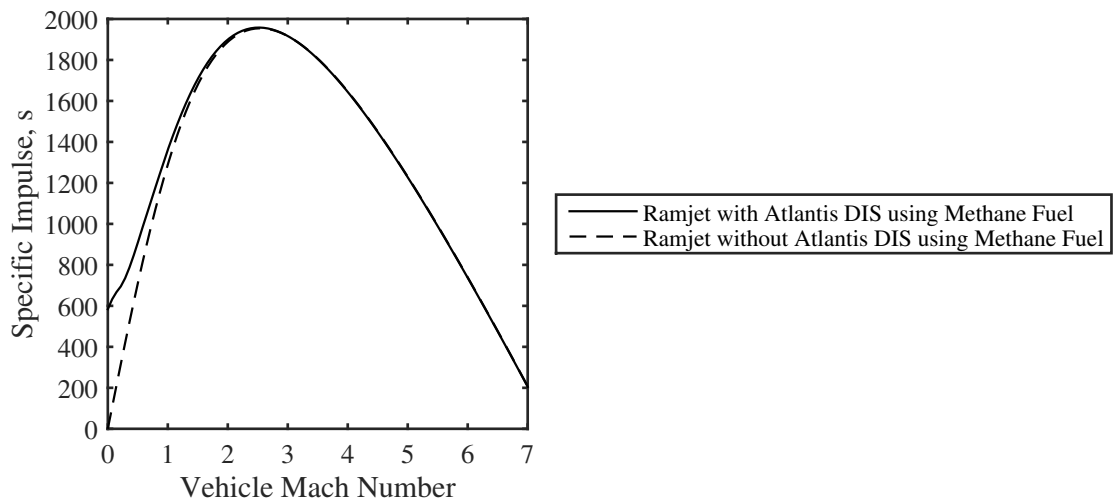

Figure 11. A comparison of Specific Impulse predicted with the method outlined for a moving vehicle with and without the AIS using a Methane fuel jet, $J P R=5.83, M a_{f}=2.0$, and $E R=1$.

Figures 8, 9, 10, and 11 represent the specific impulse curves for an engine using Kerosene, Ethylene, Propane, and Methane respectively, for fuel. For each case the value of $E R$ was fixed at one, $J P R$ was chosen as to maximize static specific impulse, and an optimized value of heat transferred was determined for each velocity. In addition to calculating the $I_{s p}$ of a ramjet utilizing the AIS, the $I_{s p}$ of an ideal ramjet without the AIS based on the same inlet conditions and maximum combustion temperature was calculated. Typically the ideal case neglects the impact of the fuel mixing with the air, as the mass fraction of fuel in a stoichiometric mixture is around 0.067 for most hydrocarbons. ${ }^{10}$ However, as the mixing of the gas streams is necessarily taken into account for the AIS, they are also being considered for the Ramjet in this case. It can be seen that in some cases the engine with the AIS maintains a greater specific impulse up to a vehicle Mach number of 2.5 , before the two lines become indistinguishable. The peak value of specific impulse, and the value at zero velocity vary significantly between the different fuels considered, this is primarily due to the unique heat of combustion, and value of $M A F_{\text {stoichiometric }}$ for each fuel.

\section{Conclusion}

Overall engine performance was quantified based on specific impulse across a range of vehicle Mach numbers for an ideal ramjet both with and without the implementation of the AIS. Figures 8 to 11 show for a variety of fuels that a significant improvement in terms of $I_{s p}$ can be seen at low Mach numbers. This is especially important at zero velocity, where the traditional ramjet has a specific impulse of zero. Through this study it can be seen that theoretically the AIS provides a means of bridging the gap between zero velocity, and the point at which a the traditional ramjet operation is feasible. Additionally, it provides a boost in performance up to Mach 2.5, with no detrimental impacts on performance beyond that point. It should be noted however, that this study did not take into account any drag impacts associated with the AIS, the implementation of such a system would likely alter performance, particularly at high vehicle 
velocities. The AIS could provide an simple and inexpensive alternative to the traditional turbojet for the initial acceleration of any ramjet propelled vehicle. These results were based on a control volume analysis performed on the AIS, which provided a simple method for the prediction of the fuel-air mixture properties exiting the AIS for both static and dynamic operation. The prediction model outlined was compared with the results of 17 simplified CFD simulations, and showed excellent agreement in terms of stagnation temperature and stagnation pressure as seen in Fig. 4 and 5. Due to the form of the solution, some unique characteristics were found. It was determined that for an array of input parameters, there will be two real solutions in terms of downstream mixture velocity. A means of determining which solution is physically most correct is provided. As well, it was found that there is a limit on increasing the mass of air-to-fuel ratio at the point where the mixture Mach number is equal to one, any further increase will result in a non-real solution.

\section{Future Work}

The primary focus of future work on this project will be the conduction of an exergetic analysis of the Atlantis Intake System to evaluate second law efficiency. Improving the models used for the pre-heating of the fuel, and the burning of the fuel within the combustion chamber will also be considered.

\section{Acknowledgments}

The financial support of this project was provided in part by Atlantis Research Labs, Natural Sciences and Engineering Research Council of Canada (NSERC) and Alberta Innovates Technology Futures (AITF).

\section{References}

\footnotetext{
${ }^{1}$ Robert Q. Riley Enterprises, "Plans and Instructions for Building the Gluhareff G8-2 Pressure Jet Engine," Tech. rep., Robert Q. Riley Enterprises, Pheonix, Arizona, 1998.

${ }^{2}$ Barrett, R. and Gluhareff, I., "Gluhareff Pressure Jet: Past, Present and Future," 46th AIAA Aerospace Sciences Meeting and Exhibition, Reno, Nevada, 2008.

${ }^{3}$ Brilliant, H., Fortson, M., Hess, D., and Torosian, A., "Further Evaluation of a Gluhareff Pressure Jet," Tech. rep., Air Force Academy Aeronautics Digest.

${ }^{4} \mathrm{Zhu}$, Y., Cai, W., Wen, C., and Li, Y., "Simplified ejector model for control and optimization," Energy Conversion and Management, Vol. 49, 2008, pp. 1424-1432.

${ }^{5}$ Huang, B. J., Chang, J. M., Wang, C. P., and Petrenko, V. A., "1-D analysis of ejector performance," International Journal of Refrigeration, Vol. 22, 1999, pp. 354-364.

${ }^{6}$ Liao, C., Gas Ejector Modeling for Design and Analysis, Ph.D. thesis, Texas A\&M University, 2008.

${ }^{7}$ John, J. E. and Keith, T. G., Gas Dynamics, Pearson Prentice Hall, Upper Saddle River, New Jersey, third edit ed., 2006.

${ }^{8}$ Bernstein, H., "Effects of Parallel-Jet Mixing on Downstream Mach Number and Stagnation Pressure with Applications to Engine Testing in Supersonic Tunnels," National Advisory Committee for Aeronautics, 1953.

${ }^{9}$ Cengel, Y. A. and Boles, M. A., Thermodynamics: An Engineering Approach, 2002.

${ }^{10}$ Spakovsky, Z. S., "Thermodynamics and Propulsion," 1999.

${ }^{11}$ FLSmidth, "Gases - Specific Heat Capacities and Individual Gas Constants," 2014.

${ }^{12}$ Arisman, C., Johansen, C., Galuppo, W., and McPhail, A., "Nitric Oxide Chemistry Effects in Hypersonic Boundary Layers," 43rd AIAA Fluid Dynamics Conference, 2012.

${ }^{13}$ Wilkes, J., Danehy, P., and Nowak, R., "Fluorescence Imaging Study of Transition in Underexpanded Free Jets," ICIASF 2005 RecordInternational Congress onInstrumentation in AerospaceSimulation Facilities, 2005.

${ }^{14}$ Ricou, F. P. and Spalding, D. B., "Measurements of entrainment by axisymmetrical turbulent jets," Journal of Fluid Mechanics, Vol. 11, 1961, pp. 21.
} 